RESEARCH PAPER RP858

Part of Journal of Research of the National Bureau of Standards, Volume 16, February 1936

\title{
A STANDARD SOURCE OF ULTRAVIOLET RADIATION FOR CALIBRATING PHOTOELECTRIC DOSAGE INTENSITY METERS
}

\author{
By W. W. Coblentz and R. Stair
}

\section{ABSTRACT}

The advantages and disadvantages of sources emitting either a continuous or a discontinuous spectrum are considered in connection with the use of such lamps as a standard of ultraviolet radiation.

It is shown that because of the high intensity and favorable distribution of the ultraviolet emission lines at 2967 to $3132 \mathrm{~A}$, the quartz mercury-arc lamp is well adapted for standardizing photoelectric ultraviolet dosage intensity meters in absolute units.

A description is given of the installation of a commercial 110-volt vertical quartz mercury Uviarc lamp, and of methods of calibrating its ultraviolet output in absolute units.

By calibrating photoelectric ultraviolet intensity meters against a laboratory standard, a great reduction is effected in equipment required for field measurements of ultraviolet solar radiation.

\section{CONTENTS}

I. Introduction

1. The quartz mercury-arc lamp versus the tungsten-filament lamp as a standard source of ultraviolet radiation

2. Tests of the emission line $2967 \mathrm{~A}$ as a standard source of

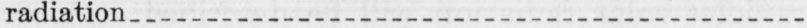

II. Description of the quartz mercury-arc lamp

III. Performance tests__.

IV. References and notes......

\section{INTRODUCTION}

The evaluation, in absolute units, of the energy in a given spectral band of radiation, by a filter method of radiometry involves two measurements: (1) the spectral quality (the spectral-energy distribution), and (2) the total intensity (the radiant flux) within and adjacent to the band of wave lengths under investigation.

Based upon the report [1] ${ }^{1}$ of the favorable performance of a balanced ("differential") thermopile and filter radiometer [2, 3], at the Copenhagen Meeting in 1932, the International Committee on Measurement and Standardization of Ultraviolet Radiation for Use in Medicine recommended to the International Congress on Light the evaluation of ultraviolet-radiation stimuli on a physical (radiometric) basis, in absolute units, by means of a nonselective radiometer (thermopile) and filters [4].

\footnotetext{
1 Numbers in hrackets refer to references and notes at the end of this paper.
} 
The thermopile and filter apparatus is essentially a primary standard of evaluation of ultraviolet radiation [2, 3, 13]. For field work the writers have recently devised a portable ultraviolet meter, which consists of a photoelectric cell, balanced amplifier, and microammeter [5]. This type of ultraviolet meter requires standardization either against the thermoelectric meter [2], which initially was calibrated against a standard of radiation or against an absolute thermopile [7], in order to standardize the microammeter reading in absolute value.

Heretofore the writers have calibrated the photoelectric ultraviolet meter directly against the type of source (the sun) under investigation, by making simultaneous measurements with the thermoelectric meter [2,3] which initially was calibrated in absolute value against a standard of radiation $[6,8]$; the photoelectric cell and filters being used to determine the spectral quality of the source (the sun) at the time of making the energy measurements with the balanced thermopile.

Without the use of complicated auxiliary apparatus the thermopile (with filters) is not sufficiently sensitive, and, hence, not well adapted for determining the spectral quality of the short wave length ultraviolet radiation of the sun; but it is the best instrument to evaluate the energy in absolute value.

Hence, to simplify the equipment for field work such as was conducted by one of the writers (W. W. C.) in Arizona, and more recently at San Juan, P. R. (where prelminary tests [9] of this method have already been made), and to simplify the computations, a photoelectric ultraviolet-intensity meter [5] is used to determine both the spectral quality and the total intensity in a given band of wave lengths of ultraviolet in sunlight.

The problem, therefore, was to devise a laboratory method of calibrating the photoelectric meter (the microammeter-scale reading) in absolute units, so that the response will be a measurement, in absolute value, of the ultraviolet intensity in sunlight [14].

In a forthcoming paper two closely agreeing methods of evaluation of ultraviolet solar radiation will be described: (1) by means of a balanced thermopile (with filters), which is calibrated in absolute value against a standard of thermal radiation $[6,8]$, and (2) by means of a photoelectric cell of titanium, calibrated in absolute value against a standard of ultraviolet radiation.

This amounts to a calibration of the photoelectric cell against a lamp in the laboratory, instead of calibrating it against the sun as a source (by making closely simultaneous measurements with the balanced thermopile, and the photoelectric meter) which, as already mentioned, was the previous procedure.

The manner of calculating the data, which is somewhat complex, will be described in the above-mentioned forthcoming paper on the evaluation of ultraviolet radiation in sunlight, for which purpose the whole scheme was devised.

The object of the present communication is to describe a convenient standard source of ultraviolet radiation, which consists of a vertical mercury-in-quartz arc lamp that is readily obtainable in commerce at a price that is not prohibitive, and which, with careful use, promises to have a long life. 


\section{THE QUARTZ MERCURY-ARC LAMP VERSUS THE TUNGSTEN- FILAMENT LAMP AS A STANDARD SOURCE OF ULTRAVIOLET RADIATION}

The following are some of the advantages in using the quartz mercury arc as a standard source of ultraviolet radiation; which source is particularly applicable for calibrating photoelectric cells used in evaluating the ultraviolet radiation, of wave lengths $3132 \mathrm{~A}$ and shorter, used for therapeutic purposes:

(1) The ultraviolet radiation of the so-called hot quartz mercury lamp is emitted in a few, conveniently distributed, intense emission lines, superposed upon a weak background of continuous spectral emission that causes little or no difficulty in correcting for spectral purity.

(2) The relative spectral intensities of these emission lines, as a function of the temperature, the energy input, and the aging of the lamp, are readily determined.

(3) The quartz mercury arc is in common use for determining (a) the transmission of the spectrometer used in measuring the spectral intensities of the emission lines in absolute values, (b) the spectral transmission of the filters used in the filter method of radiometery, (c) the spectral response of the photoelectric cell, used in measuring ultraviolet radiation, and (d) the effects of ultraviolet in biological investigations.

(4) The emission line at $2967 \mathrm{~A}$, emitted by the quartz mercuryarc lamp, evaluated in absolute units, was found useful in standardizing skin sensibility, in connection with investigations of the erythemal reaction produced by different sources of ultraviolet radiation [3], and recently this emission line was used in connection with the study of the effect of environment upon the production of erythema [10].

(5) The total ultraviolet in a given spectral band can be determined in absolute units, by measuring the intensities in the individual lines in absolute value, as was done successfully by seven research laboratories [11], using different kinds of spectroradiometers, and quartz mercury-arc lamps that were interchanged between the various laboratories. If the participating laboratories had used the most modern apparatus and methods there would have resulted a still closer agreement in the measurements.

(6) The total radiation, in absolute value, included in the spectral band of wave lengths $3132 \mathrm{~A}$ and shorter (of special interest in therapy) can be accurately evaluated, as indicated by the agreement in the values obtained by summation of the intensities of the individual spectral lines, and by integrated measurements of the undispersed radiation, using a balanced thermopile and filter radiometer $[2,3,11]$.

(7) The use of a discontinuous spectrum of intense emission lines, readily permits testing the accuracy of the energy evaluation, as for example, in the studies of the germicidal action of ultraviolet radiation, conducted by Coblentz and Fulton [12], in which practically exact agreement was found between the observed and the calculated transmissions of the filters used to determine the spectral range of the germicidal action.

(8) In calibrating a modern photoelectric cell which is insensitive to wave lengths longer than about $3300 \mathrm{~A}$, and in which the short- 
wave-length response is limited (at about $2800 \mathrm{~A}$ ) by its inclosure of Corex D glass, practically only the strong emission lines at 2967, 3024 , and 3132 A are effective.

The energy value of these few lines is easily determined in absolute units, by two methods $[2,3,11]$, and from a knowledge of the spectralresponse curve of the photoelectric cell there is obtained the true scale reading of the microammeter [5] that would have resulted if the photoelectric cell had been nonselective in spectral response.

From the viewpoint of cost, a gas-filled tungsten lamp has been considered for use as a standard source of ultraviolet radiation for calibrating photoelectric ultraviolet meters. The following are some of the disadvantages in using, as a standard source of ultraviolet radiation, a lamp which emits a continuous spectrum, for example, a gas-filled tungsten lamp:

(1) The spectral intensity of the ultraviolet, particularly of wave lengths shorter than $3000 \mathrm{~A}$, emitted by a tungsten-filament lamp is low, and, hence difficult to determine accurately in absolute value.

(2) There is difficulty and uncertainty in correcting for spectral purity in reducing the observed intensities in the continuous spectrum, from the prismatic to the normal spectral distribution-a necessary procedure, since different laboratories use spectroradiometers having different dispersion and transmission constants.

(3) Owing to uncertainty in spectral purity, there is difficulty in establishing the long-wave-length limit, say at $3130 \mathrm{~A}$, of the cut-off, in integrating a spectral band of radiation such as, for example, the band of ultraviolet radiation, including for all practical purposes the wave length $3132 \mathrm{~A}$ (and shorter wave lengths), which is recognized as having a therapeutic value in healing rickets.

(4) Based upon the experience of one of the writers (W. W. C.), extending over a period of three decades, the accurate mapping of the continuous spectrum of low intensity is extremely difficult and uncertain, particularly the evaluation of the energy in a narrow spectral band, in absolute units, because of the uncertainty of the corrections for absorption in the spectrometer.

(5) The continuous spectrum is not readily adapted for making the calculations required in connection with the evaluation of the spectral response of the photoelectric cells or for checking the accuracy of the calibrations, by comparing the observed with the calculated filter transmissions.

\section{TESTS OF THE EMISSION LINE 2967 A AS A STANDARD SOURCE OF RADIATION}

For calibrating a photoelectric ultraviolet-intensity meter [5] in absolute value, the simplest procedure would appear to be to observe the microammeter-scale reading on exposure of the photoelectric cell to a strong emission line, the energy value of which is easily determined in absolute units [3]. Then the true scale reading would be obtained by dividing the observed scale reading by the efficiency of the photoelectric response for that wave length. The resulting microammeter reading would then be relatively the same as that of a spectrally nonselective radiometer.

The writers have attempted to calibrate their photoelectric ultraviolet-intensity meter [5], in absolute value, by means of homo- 
geneous radiation of wave length $2967 \mathrm{~A}$, because this emission line has a number of advantages which make it useful as a standard in erythemal and photobiological investigations $[1,3,13]$.

However, the attempt to calibrate the photoelectric ultraviolet meter [5] used in evaluating the ultraviolet in sunlight, by employing the emission line of mercury, at $2967 \mathrm{~A}$ as a standard, was not very successful, owing to the manner of construction of the photoelectric cell (the heavy-wire anode occupies the center of the field), which caused difficulties in operating the device at the exit slit of the spectroradiometer used in isolating the emission line $2967 \mathrm{~A}$.

Hence, since the radiation from the quartz mercury arc, effective on the photoelectric cells used by the writers, can be confined practically to four emission lines (at 2894, 2967, 3024, and $3132 \mathrm{~A}$, by using a filter of Corex D glass), which can be accurately evaluated in absolute units $[2,3,11]$, the writers have adopted this type of lamp as a standard source of ultraviolet radiation for calibrating photoelectric dosage intensity meters.

To attain the highest accuracy in the calibrations, the ultraviolet output of the lamp is determined with the balanced thermocouple and filters [2, 3], at the time the photoelectric measurements are made, and at the same distance from the lamp. This eliminates the difficulty of setting the photoelectric ultraviolet meter at a known distance from the lamp, and of operating the lamp at a fixed room temperature, which has a slight effect on the output at a given voltage.

Another procedure is to operate the lamp at a constant-energy input, in the burner, in an inclosure that is maintained closely at the same air temperature. In this manner a single standardization of the lamp against the balanced thermopile, at a fixed distance from the lamp, will suffice until the lamp shows signs of aging (blackening).

The reduction in intensity (aging by blackening of the part of the quartz tube surrounding the tungsten anode), and a possible unsteadiness of the radiation resulting from the constant flickering of the luminous column directly above the mercury cathode are eliminated by placing in front of the lamp a diaphragm, having an opening $30 \mathrm{~mm}$ in length, which limits the emission to the central portion of the lamp, about $30 \mathrm{~mm}$ in length.

In conclusion it is relevant to summarize the foregoing discussion by recalling that in previous solar ultraviolet measurements in the field (e. g., in Arizona) two sets of instruments were used to evaluate the ultraviolet in sunlight, (1) photoelectric cells and filters to measure the spectral quality, and (2) a balanced thermopile and filter radiometer to evaluate the total intensity of ${ }^{2}$ the ultraviolet, in absolute units.

By calibrating the portable ultraviolet intensity meter [5] against the herein-described standard source of ultraviolet radiation, it will not be necessary to carry the balanced thermopile outfit into the field. This was amply demonstrated in a recent calibration of a recording ultraviolet meter, and in an investigation of the spectral quality (ozone content) of the ultraviolet, in San Juan, P. R. [9], in which only the portable ultraviolet intensity meter was employed. Checked against the standard source, on the return, the calibration of the photoelectric cell was found unchanged; and since, as will be shown in a forthcoming paper, the evaluation of the ultraviolet in sunlight by the thermoelectric and the photoelectric methods is the same, there 
is a considerable saving in time and expense in transportation, by using only the standard portable photoelectric meter [5], with several standardized photoelectric cells in reserve.

In view of the fact that in earlier communications the writers were opposed to the use of photoelectric cells as ultraviolet meters, it is relevant to add that recent improvements have overcome the objectionable features of earlier makes of photoelectric cells, in which there was great fatigue and in which the response depended upon the applied voltage and was not proportional to the intensity. As pointed out elsewhere [14] the modern, highly evacuated photoelectric cell of titanium or cadmium, with its selective spectral response confined to wave lengths shorter than $3300 \mathrm{~A}$, in contrast with the nonselective response of the thermopile, appears to be an advantage instead of a disadvantage, at least in evaluating short wave length $(2900$ to $3300 \mathrm{~A})$ ultraviolet solar radiation. This is owing to the fact the spectralenergy curve of the sun, in the region of 3300 to $4000 \mathrm{~A}$, is not known with sufficient accuracy to calculate the energy in this spectral band, and there are no suitable filters to place over the thermopile, to completely eliminate this part of the spectrum and confine the measurements to the spectral band of wave lengths shorter than $3300 \mathrm{~A}$, which is possible with a photoelectric cell that does not respond to wave lengths longer than about $3300 \mathrm{~A}$. In recent measurements of solar ultraviolet radiation, the writers placed an especially selected filter of Corning Purple Corex glass, G 986-A, over the thermopile [2], thus reducing its spectral response principally to wave lengths shorter than about $4000 \mathrm{~A}$, and apparently increasing the accuracy of the calculations of the results. In view of the complexity of the apparatus and experimental procedure involved in making the observations, the closely agreeing results obtained by the two methods of measurement (thermoelectric and photoelectric), appear to show that the data are substantially correct.

\section{DESCRIPTION OF THE QUARTZ MERCURY-ARC LAMP}

During the past several years the writers have used several types of $d-c$ and a-c mercury-in-quartz arc lamps, operated in the horizontal and the vertical positions, for calibrating ultraviolet meters [14]. The earliest calibrations of ultraviolet meters were made with a Hanovia Luxor Model, a-c therapeutic lamp, which had a satisfactory performance but which, on account of its horizontal position, was not convenient to operate in front of a spectroradiometer.

The type of lamp adopted is the Cooper-Hewitt (General Electric Vapor Lamp Co.), 110-volt, vertical Uviarc, mercury-in-quartz arc lamp, which is the most convenient arrangement for all types of measurements, particularly with a spectroscope.

The clear part of the quartz tube, which is about $8 \mathrm{~cm}$ long between the electrodes and about $18 \mathrm{~mm}$ in diameter, is covered with a sheet of aluminum ( $\mathrm{W}$ in fig. 1) having an opening 30 by $30 \mathrm{~mm}$, which produces a symmetrical source free from the flickering noticeable over the lower mercury electrode and excludes the upper end, part of which may become blackened from the tungsten anode.

As shown diagrammatically in figure 1, the support for the quartz tube (the "burner") consists of a right-angle piece of aluminum A, attached to the nickel-plated iron rod $R$, in a support that permits 
rotation of the arc into the horizontal position, which is necessary to start the arc. Adjustable clamp S serves as a stop to place the lamp in the same vertical position after each starting of the lamp.

The lamp rests in a porcelain or Pyrex support $P$, which insulates it from the aluminum frame. The top support of the lamp is a strip

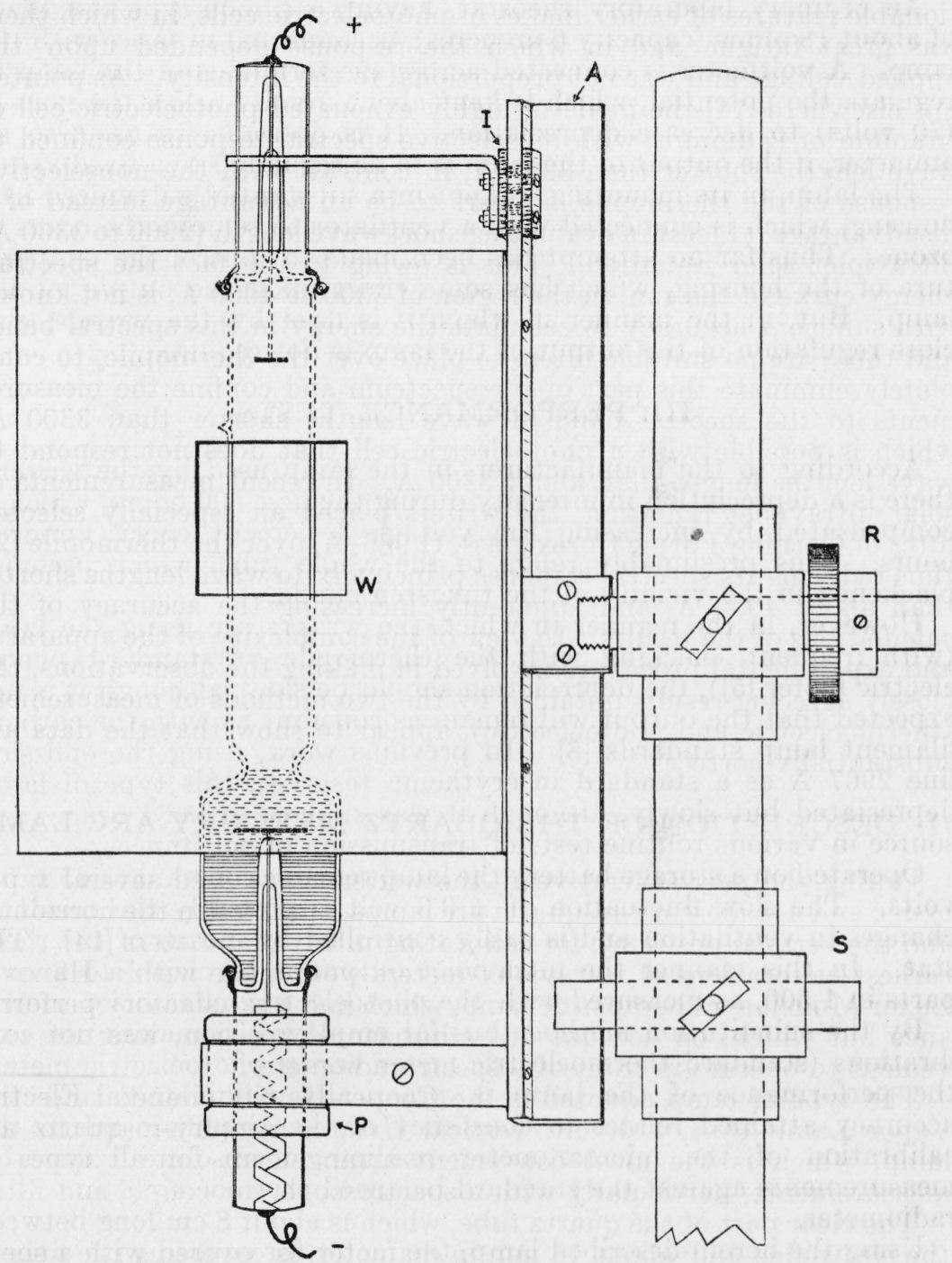

Figure 1.-Mounting of ultraviolet radiation standard.

of aluminum, which is insulated from the vertical support $\mathrm{A}$ by means of mica and asbestos, I.

The electrical connections (indicated diagrammatically) are thin pliable strips of brass, some $5 \mathrm{~cm}$ long at the free ends, which remain cool, and, hence, do not disintegrate by the heat of the electrodes. The lead-wires are soldered to these metal strips. 
An aluminum shield $\mathrm{W}$ is securely attached to the vertical support $A$, and forms a benchmark for measuring distances. With the exception of the aluminum support A, the remaining part of the lamp holder consists of a tripod, rods, and right-angle clamps, which form the equipment of the average physics laboratory.

An ordinary laboratory rheostat, having a ribbon-type resistance of about $18 \mathrm{ohms}$ (capacity 6 amperes), is connected in series with the lamp. A voltmeter is connected across the terminals of the lamp to regulate the potential, which is kept about 5 volts below the normal (70 volts) to decrease depreciation. It is desirable to use also an ammeter, if the output of the lamp is to be regulated to a fixed value.

The lamp, in its mounting, is kept in a substantial galvanized-iron housing, which is connected with a ventilator to remove the odors of ozone. Thus far no attempt has been made to control the temperature of the housing, which has some effect upon the output of the lamp. But, in the manner in which it is used by the writers, this close regulation of the output of the lamp is not required.

\section{PERFORMANCE TESTS}

According to the manufacturer, in the lamp used by the writers, there is a depreciation in intensity during the first 500 hours, which is compensated by increasing the voltage 3 percent every hundred hours. This presumably refers to the whole tube, which becomes blackened in the vicinity of the tungsten anode.

However, in the manner in which the writers are using the lamp (with frequent checking with the thermopile or standard photoelectric meter [5]), the depreciation should be slow; although it is not expected that the output will remain as constant as with the carbonfilament lamp standards [8]. In previous work, using the emission line $2967 \mathrm{~A}$ as a standard in erythema tests [4], this type of lamp depreciated but slowly, although it was subject to hard usage as a source in various routine tests of transmission of substances.

Operated on a storage battery the lamp remains constant at $65 \pm 0.3$ volts. The slow fluctuation of \pm 0.3 volt is probably the result of changes in ventilation and is easily controlled by means of the rheostat. In this manner the ultraviolet output is regulated to 2 or 3 parts in 1,500, as measured with the photoelectric ultraviolet meter.

By the substitution method thus far employed in making the calibrations (standard thermoelectric meter versus photoelectric meter) the performance of the lamp is practically eliminated, and the accuracy attained relates to constancy of the amplifier and to the calibration of the microammeter reading in absolute value, by measurements against the standard balanced thermocouple and filter radiometer.

Using the herein-described lamp, the factor for equating the microammeter reading into absolute units (microwatts per $\mathrm{cm}^{2}$ ) using photoelectric cell Ti-1, determined on different days, was 9.33 and 8.97, respectively, the whole apparatus having been dismounted (the window of the photoelectric cell was freed from dust) and reset in the meantime. The dust particles, which became attached by an electric charge on the glass, would reduce the transmission by 1 or more percent, and on removal would decrease the factor 9.33 to a lower value, as observed. Hence, the deviation of 2 percent from the 
mean value (9.15) is ascribable partly to dust adhering to the window of the photoelectric cell. The average of four calibrations, made between January 22 and December 16, 1935, using two Uviarc lamps, is 9.06 - a difference of only 1 percent from the most recent and presumably the most reliable determinations.

In this connection, it is to be noted that there is great difficulty in keeping the photoelectric cells and filters perfectly free from electrically charged particles of dust, especially when working in a dry climate, at high altitudes.

The high accuracy attainable in the calibration of photoelectric ultraviolet meters in the laboratory is entirely illusory. It has no meaning in attempting to evaluate the constantly changing intensity of the ultraviolet in sunlight, for which this double check in the calibration was devised.

The agreement in the absolute values of the ultraviolet intensities in sunlight, under various atmospheric conditions, as deduced by the two above-described methods of measurement (photoelectric and thermoelectric) will be described in a forthcoming paper.

\section{REFERENCES AND NOTES}

[1] W. W. Coblentz, Comp. Rend. du IIe Congrès International de la Lumière; biologie, biophysique, therapeutique, p. 322 (published by Engelsen and Schroeder, Copenhagen, 1932); reprinted in Strahlentherapie 50, 179 (1934).

[2] W. W. Coblentz, R. Stair, and J. M. Hogue, BS J. Research 7, 723 (1931) RP370.

Tests of balanced thermopile and filter in evaluating ultraviolet solar radiation; integration of ultraviolet radiation by spectroradiometric, and by thermocouple and filter methods; calibration of photoelectric cell in absolute units.

[3] W. W. Coblentz, R. Stair, and J. M. Hogue, BS J. Research 8, 759 (1932) RP450.

Description of the evaluation of ultraviolet in different sources by means of a balanced thermocouple and filter radiometer, used as a dosage intensity meter; p. 769 enumerates the advantages in using homogeneous radiation of wave length 2967 A as a standard source for radiometric and physiological (erythema) tests.

[4] W. W. Coblentz, Science 76, 412 (1932).

Report on the Copenhagen meeting of the Second International Congress on Light.

[5] W. W. Coblentz and R. Stair, BS J. Research 12, 231 (1934) RP647.

Description of a portable ultraviolet intensity meter consisting of a photoelectric cell, balanced amplifier, and microammeter.

Considering the progress described in the present paper in producing such a standard, the statement (on p. 232) that there is as yet no (simple) standard of ultraviolet radiation for accurately testing the constancy of photoelectric cells requires qualification.

[6] W. W. Coblentz, Bul. BS 11, 87 (1914) S227; 12, 580 (1916) S262.

Investigation and preparation of standards of radiation for calibrating radiometers (thermopiles) in absolute value.

[7] W. W. Coblentz, Bul. BS 9, 51 (1912) S188.

Tests of the Callendar radiobalance. W. W. Coblentz, Bul. BS 11, 157 (1914) S229; W. W. Coblentz and W. B. Emerson, Bul. BS 12, 503 (1916) S261; Gerlach, Ann. Physik 38, 1 (1912).

Studies of angstrom pyrheliometers and thermopiles for measurement of radiation in absolute value.

[8] W. W. Coblentz and R. Stair, BS J. Research 11, 79 (1933) RP578.

Verification of standards of radiation.

[9] W. W. Coblentz and R. Stair, J. Research NBS 15, 123 (1935) RP816.

Effect of latitude on ultraviolet solar intensities.

[10] H. Danneel, Strahlentherapie, 53, 171 (1935).

Used the emission line at $2967 \mathrm{~A}$ to standardize erythema in testing the effect of environment upon cutaneous sensibility to ultraviolet radiation.

[11] Report of the Illum. Eng. Soc. Subcommittee on the Measurement and Evaluation of Ultraviolet Radiation, Trans. Illum. Eng. Soc. 28, 864 (1933). 
[12] W. W. Coblentz and H. R. Fulton, BS Sci. Pap. 19, 641 (1924) S495.

[13] W. W. Coblentz, Strahlentherapie 50, 487 (1934); Am. J. Roentgenol. Radium Therapy 33, 793 (1935).

Considerations relative to the evaluation of ultraviolet radiation in absolute units.

[14] W. W. Coblentz, Puerto Rico J. Pub. Health Trop. Med. 11, 1 (1935).

Discussion of the problem of evaluating ultraviolet radiation for use in medicine. An outline is given of the newly developed method of evaluating ultraviolet solar radiation, using a standard of ultraviolet radiation for calibrating the photoelectric dosage intensity meter in absolute units.

Washington, December 16, 1935. 